\title{
Isolation of Cellulose Nanofibers: Effect of Biotreatment on Hydrogen Bonding Network in Wood Fibers
}

\author{
Sreekumar Janardhnan and Mohini Sain \\ Department of Chemical Engineering \& Applied Chemistry, University of Toronto, 200 College Street, Toronto, ON, Canada M5S 3E5 \\ Correspondence should be addressed to Sreekumar Janardhnan, s.janardhnan@utoronto.ca
}

Received 10 March 2011; Accepted 3 May 2011

Academic Editor: Susheel Kalia

Copyright ( $) 2011$ S. Janardhnan and M. Sain. This is an open access article distributed under the Creative Commons Attribution License, which permits unrestricted use, distribution, and reproduction in any medium, provided the original work is properly cited.

\begin{abstract}
The use of cellulose nanofibres as high-strength reinforcement in nano-biocomposites is very enthusiastically being explored due to their biodegradability, renewability, and high specific strength properties. Cellulose, through a regular network of inter- and intramolecular hydrogen bonds, is organized into perfect stereoregular configuration called microfibrils which further aggregate to different levels to form the fibre. Intermolecular hydrogen bonding at various levels, especially at the elementary level, is the major binding force that one need to overcome to reverse engineer these fibres into their microfibrillar level. This paper briefly describes a novel enzymatic fibre pretreatment developed to facilitate the isolation of cellulose microfibrils and explores effectiveness of biotreatment on the intermolecular and intramolecular hydrogen bonding in the fiber. Bleached Kraft Softwood Pulp was treated with a fungus (OS1) isolated from elm tree infected with Dutch elm disease. Cellulose microfibrils were isolated from these treated fibers by high-shear refining. The $\%$ yield of nanofibres and their diameter distribution $(<50 \mathrm{~nm})$ isolated from the bio-treated fibers indicated a substantial increase compared to those isolated from untreated fibers. FT-IR spectral analysis indicated a reduction in the density of intermolecular and intramolecular hydrogen bonding within the fiber. X-ray spectrometry indicated a reduction in the crystallinity. Hydrogen bond-specific enzyme and its application in the isolation of new generation cellulose nano-fibers can be a huge leap forward in the field of nano-biocomposites.
\end{abstract}

\section{Introduction}

Cellulose is the most important constituent of the cell wall and forms a framework around which all other cell wall polysaccharides like hemicellulose, lignin, and pectin are deposited during the plant cell growth [1].

1.1. Cellulose Microfibrils. Cellulose microfibrils are a selfassembly of cellulose chains that are synthesized by plasma membrane, which through a regular network of inter- and intramolecular hydrogen bonds are organized into perfect stereoregular configuration called microfibrils. Each chain is stabilized by intrachain hydrogen bonds formed between the pyranose ring oxygen in one residue and the hydrogen of the $\mathrm{OH}$ group on $\mathrm{C} 3$ in the next residue $\left(\mathrm{O} 5 \cdots \mathrm{H}-\mathrm{O}^{\prime}\right)$ and between the hydroxyls on $\mathrm{C} 2$ and $\mathrm{C} 6$ in the next residue (O2$\left.\mathrm{H} \cdot \mathrm{O}^{\prime}\right)[2]$.

Microfibrils are generated in the laboratory through a combination of high-energy refining in a PFI mill and subsequent cryocrushing under the presence of liquid nitrogen [3].

The elementarization of natural fibres into thier elementary cellulosic constituents like microfibrils is gaining wider attention due to their high strength and stiffness [4], high reinforcing potential [5], and their biodegradability and renewability.

1.2. Application. Numerous high-end potential applications for cellulose microfibrils are currently being explored. Cellulose microfibrils can be used to make ultralight composite materials (e.g., film). Microfibrils are being used to reinforce biopolymers like polylactic acid and starch-based polymers for packaging materials that are $100 \%$ biodegradable. Cellulose-based nano-biocomposites are also gaining considerable attention in various structural application in auto- and aerospace industry. Cellulose microfibrils give considerable toughness and strength to traditional paper products even in small quantities. 
1.3. Challenges. Utilization of cellulose nano-fibers in composites is faced with three major challenges-isolation (high energy requirement), interfacial compatibility, and chemistry and dispersion of cellulose nano-fibers in polymer matrix.

The very first approach to utilizing hydroxyl chemistryspecific enzymes to facilitate the isolation of cellulose microfibrils from wood plant cell wall was successfully demonstrated in [6]. Hydrogen bond cleaving enzymes from a specific fungus was used to bring about internal defibrillation in the fibre. This paper briefly explores the mechanism behind the action of a specific enzyme in bringing about internal defibrillation in the fibre cell wall which is important in addressing the high-energy requirement associated with cellulose nanofibre isolation from plant fibres.

1.4. Enzyme Technology in Isolation of Cellulose Microfibrils. Although enzymes have been widely used to modify cellulosic fibres for various applications enhancement [7-9] and drainage [10-13], there has not been any research effort to understand and utilize the enzyme-fiber interaction at the fiber molecular level. An understanding of the chemistry at this level and its exploitation to isolate high-strength nanofibers from plant cell wall in an economical manner will be a huge step towards their commercial scale utilization in various applications.

\section{Materials and Methods}

\subsection{Materials}

2.1.1. Wood Fibre. Bleached Kraft Softwood Pulp, northern black spruce, was used as the starting material for the isolation of microfibrils. The fiber has a cellulose content of $86 \%$ and hemicellulose content of $14 \%$.

2.1.2. Fungus. OS1, a fungus isolated in our laboratory from elm tree infected with Dutch Elm disease, was used as the source of enzyme for the fibre treatment.

\subsection{Methods}

2.2.1. Biotreatment. Bleached kraft fibre was soaked overnight and thoroughly disintegrated in 2 liters of water and autoclaved for 20 minutes. $200 \mathrm{~mL}$ of OS1 fungal culture was added to a 2-liter fibre suspension (containing $24 \mathrm{~g}$ dry fibre) in a sterile flask with $10 \mathrm{~g} / \mathrm{L}$ of sucrose and $2 \mathrm{~g} / \mathrm{L}$ of yeast extract to support the fungal growth. The fungus was left to grow on the fibres at room temperature for a period of three to four days with slow agitation. The fibres are autoclaved after their respective treatment time and washed for further processing to cellulose nano-fibers.

2.2.2. High-Shear Refining. The biotreated fibres were passed through a PFI (Paperindustriens Forkninginstitutt, Oslo, Norway) high-shear refiner to further affect internal defibrillation. 24 grams of fiber at $10 \%$ consistency was charged into the PFI mill and sheared for 100,000 revolutions to affect internal defibrillation in the fibre.

\subsection{Fiber Characterization}

2.3.1. Transmission Electron Microscopy (TEM). Phillips CM 201 model was used for this study. The transmission electron microscopy (TEM) images were used to understand the surface morphology and diameter distribution of the treated fibres and cellulose microfibrils isolated.

2.3.2. FI-IR Spectroscopy. The hydrogen bond type and density associated with the fibre were investigated using FTIR spectroscopy.

Dried cellulose samples over phosphorus pentoxide in a desiccator were made into a pellet with Potassium Bromide $(\mathrm{KBr})$ powder $(1: 5$, cellulose: $\mathrm{KBr})$ and analyzed by FT-IR spectroscopy. The FT-IR spectra (256 scans, $4 \mathrm{~cm}^{-1}$ ) were determined by the diffuse reflectance method (DRIFT) using a Bruker Tensor 27 spectrometer.

There are many powerful tools which may be used to investigate cellulose chemistry and its structure: X-ray, electron diffraction and microscopy, FTIR, FT-Raman and solid-state MNR of which FT-IR is one of the most versatile techniques to study the hydrogen bond formation. Using FT-IR, Fengel $[14,15]$ analyzed the hydroxyl absorption bands by deconvoluting the spectra of cellulose. Michell $[16,17]$ used the 2nd derivative mode of the FT-IR spectra to improve its resolution considerably. Sugiyama et al. [18] and Michell [19] used FT-IR spectral data to establish and confirm the findings of Wiley and Atalla [20], that the crystalline dimorphism of native cellulose, cellulose $I_{\alpha}$ and $I_{\beta}$ were considered to differ in their hydrogen bonding rather than in their conformation. Tashiro and Kobayashi used IR spectra to determine the $\mathrm{O}-\mathrm{H}$ stretching frequencies due to intra- and intermolecular hydrogen bonds in cellulose I and II.

Fourier Self-Deconvolution. All FT-IR spectra are baseline corrected and compensated for $\mathrm{CO}_{2}$ and $\mathrm{H}_{2} \mathrm{O}$ before any manipulation.

The aim of Fourier self-deconvolution is to enhance the apparent resolution of a spectrum or to decrease the line width. Fourier self-deconvolution (FDS) assumes that the spectrum to be measured consists of well-resolved lines which have been convoluted by the same type of line broadening function (LBF). If the shape and width of the LBF are known, its effects can be arithmetically excluded from the spectra. In general, the deconvolution corresponds to a multiplication of the interferogram $I(x)$ using the exp $(a * x)$ deconvolution function for Lorentzian and $\exp (a * x * x)$ for Gaussian shapes. The deconvolution factor is the maximum value of these functions at the end of the interferogram. To avoid the increase of noise caused by the deconvolution, a Blackman-Harris apodization is simultaneously performed on the spectra.

Curve Fit. Curve fitting procedure was used to calculate single components in a system of overlapping bands. A model consisting of an estimated number of bands is essen-tial before the fitting calculation is started. The IR absorption bands for the $\mathrm{OH}$ stretchingregions were 
deconvoluted before the curve fitting procedure. To improve the calculation, the peaks need to be resolved and their number, position, and areas determined. The number of peaks and their position were determined by the point at which the second derivative of the spectrum contained peaks of interest. The position of the deconvoluted bands can give us information about the nature and type of $\mathrm{OH}$ functionality.

\section{Results and Discussion}

3.1. Effect of OS1 Pretreatment of Fibres on Cellulose Nanofibres Yield and Fibre Diameter Distribution. As mentioned earlier, one of the major challenges impeding the isolation of cellulose nanofibres on a sizable scale for any application is the high-energy requirement associated with neutralizing the predominating hydrogen bonds between the cellulose microfibrils and also between microfibrils and hemicellulose. The interfibrillar hydrogen bonding energy has to be overcome in order to separate the microfibres into individual entities. This association energy for cellulose ranges between 19 and $21 \mathrm{MJ} / \mathrm{kg} \mathrm{moL}$, with $20 \mathrm{MJ} / \mathrm{kg} \cdot \mathrm{moL}$ being used as an average value in most cases.

OS1 fungus was used for the fibre treatment and is based on our prior knowledge of their effect on hemp fibresits capacity to degrade and probably hydrolyze the cellulose [21].

\subsubsection{Yield and Diameter Distribution of Cellulose Nanofibres.} The effect of bio-treatment on the yield of cellulose nanofibres and their diameter distribution is detailed in an earlier publication by Janardhnan and Sain [6].

Fibres treated with OS1 were PFI refined and the number average diameter distribution of these refined fibres for 4day treatment is detailed in Figure 1. Cellulose nanofibre diameter distribution showed a significant shift towards the lower diameter range with the maximum yield of fibres occurring between 50 and $75 \mathrm{~nm}$ for the 4-day biotreated fibres while the maximum yield for the untreated fibre occurred between 100 and $250 \mathrm{~nm}$.

The separation of elementary fibres takes place to a good extent with biotreated fibres while PFI refining seems to have a less fibril separation effect on untreated fibers. The isolated nano-fibers from biotreated and untreated fibers showed distinct morphological differences. There is a distinct separation of elementary fibers into individualized nanofibres for biotreated fibers compared to limited microfibrillation and separation for the untreated fibres after high-shear refining.

3.2. Effect of Enzymatic Pretreatment on the Hydroxyl Chemistry of Cellulose Fibre. Having established the fact that the biotreatment can bring about a certain degree of internal defibrillation in the treated wood fibre, it is important to substantiate and validate the possible mechanism in this effect. It is assumed that the internal defibrillation is a result of the cleavage of hydrogen bonds between elementary cellulose fibrils. Analysis of the FTIR spectra of biotreated

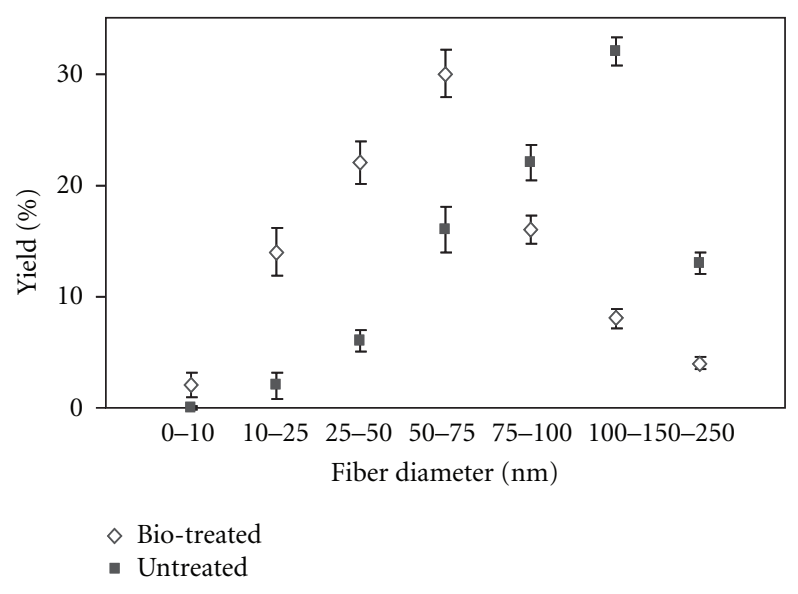

FIGURE 1: Effect of OS1 fungal treatments on number average diameter distribution of nanofibres after PFI refining for a 4-day treatment.

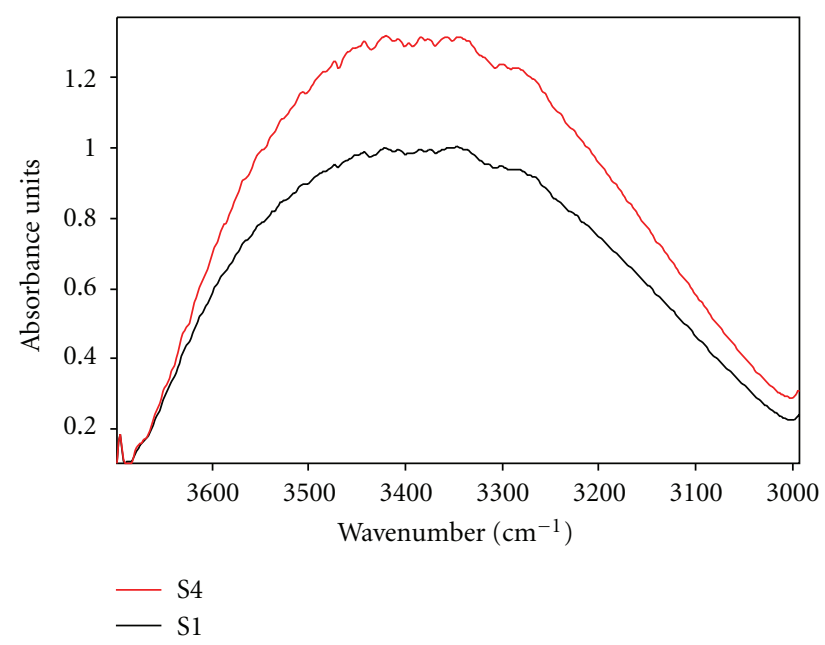

FIgure 2: FT-deconvoluted $\mathrm{OH}$ bands $\left(3600\right.$ to $3200 \mathrm{~cm}^{-1}$ ); S1: biotreated; S4: untreated wood fiber.

and untreated fibers is done here to support and validate this assumption.

FT-IR spectra of the untreated fibres and the biotreated fibres are FT-deconvoluted to improve the resolution of the $\mathrm{OH}$ stretching band and evaluated by curve fitting to determine the contributions of the integral intensities of each of the components to the total intensity of the whole band. Normalized FT-IR spectra of 4-day biotreated and untreated cellulose fibres are shown in Figure 2. The spectrum was obtained with equal amount of cellulose fibres and $\mathrm{KBr}$ to contain relative quantitative information about the $\mathrm{OH}$ functionality and their interaction in the sample.

The band at $2900 / \mathrm{cm}$, which corresponds to C-H stretching vibration, is relatively unaffected by the degree of bio-treatment or other variables. Area under the peak $2900 / \mathrm{cm}$ is identified in Table 1. Thus, the area under this $2900 / \mathrm{cm}$ band was selected to serve as an internal standard for comparison. 
TABle 1: Change in IR Index of wood fire with enzymatic treatment.*

\begin{tabular}{lccc}
\hline $\begin{array}{l}\text { Enzyme } \\
\text { treatment }\end{array}$ & $\begin{array}{c}\text { OH peak area } \\
2997-3742 / \mathrm{cm}\end{array}$ & $\begin{array}{c}2900 / \mathrm{cm} \\
\text { Peak area }\end{array}$ & $*$ Hydrogen bonds \\
\hline S1 & 624.958 & 69.92 & 8.94 \\
S4 & 681.227 & 70.52 & 9.66 \\
\hline
\end{tabular}

S1: 4-day OS1 treatment; S4: untreated.

* Calculated by relative peak area with $2900 / \mathrm{cm}$ as internal standard.

3.2.1. Relative Intensity of Hydrogen Bond Network. Hydroxyl $\left(\mathrm{OH}^{-}\right)$is the main functional group in cellulose, having various hydrogen bonding acceptors for the formation of hydrogen bonds. The untreated wood fibre sample, S4, shows 1.3 IR absorption units compared to the IR absorption unit of 0.7 with biotreated fibers as shown in Figure 2. A comparison of areas under the respective peaks is shown in Table 1. The absorption bands associated with $\mathrm{OH}$ stretching vibration in the cellulose samples indicate a difference in their absorption level suggesting a difference in the relative availability of $\mathrm{OH}$ in the samples analyzed. The difference in this IR absorption levels between the biotreated and untreated cellulose could be attributed to the difference in the density of hydrogen bonding in the fibre. Internal defibrillation that is happening in the fibres due to bio-treatment can be due to cleavage of hydrogen bonding that exists between the elementary cellulose fibres within the fibre.

3.2.2. Nature of Hydrogen Bonds. To better understand the effect of bio-treatment on the $\mathrm{OH}$ functionality and the hydrogen bonding type and pattern in the cellulose fibre, the $\mathrm{OH}$ bands associated with $\mathrm{S} 1$ and $\mathrm{S} 4$ are deconvoluted and curve fitted to individual bands that have a major contribution to the overall intensity of the $\mathrm{OH}$ band. To resolve this thought further, the peaks need to be resolved and their number and positions accurately determined. The major 2 nd derivative peak in the region of interest (3600 to $3200 \mathrm{~cm}^{-1}$ ) associated with FT-deconvoluted $\mathrm{OH}$ band was used for this purpose.

The peaks of interest are picked from the FTdeconvoluted bands of S1 and S4, and a model is set up to run the curve fitting procedure. Calculations were repeated until a best fit was obtained with rms error less than 0.01 .

Investigators have established that the two bands between 3500 and $3400 / \mathrm{cm}$ are derived from the intramolecular hydrogen bonds between $\mathrm{OH}(3)$ and $\mathrm{O}(5)$ and that the bands between 3400 and $3100 / \mathrm{cm}$ are derived from intermolecular hydrogen bonds.

Curve-fitted $\mathrm{OH}$ bands (Figures 3 and 4) based on the peaks of interest picked, were found to provide more insight into the individual peaks that contributed to the broad $\mathrm{OH}$ band. FT-IR absorption characteristic bands associated with $\mathrm{S} 4$-untreated fibre in the $\mathrm{OH}$ stretching region demonstrated a marked difference from that of the peaks for S1 4-day OStreated fibres.

Intermolecular Hydrogen Bonds. A careful observation of the IR absorption characteristic curves for biotreated fibre and

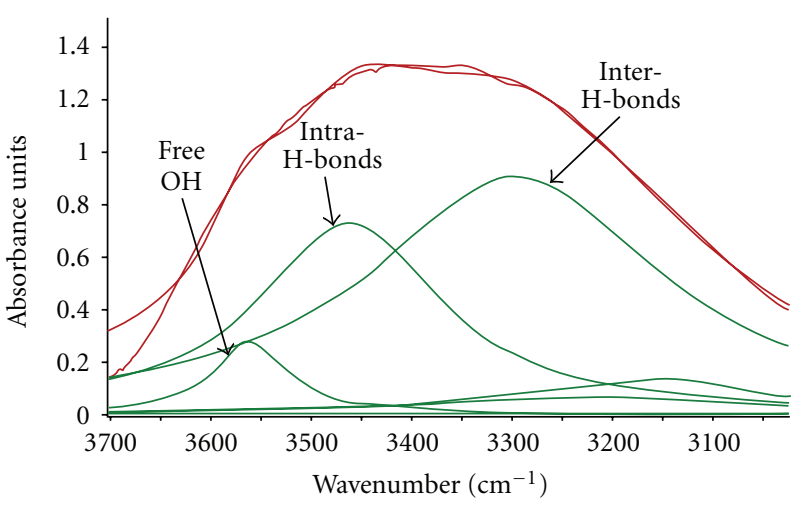

Figure 3: Curve fitting and peak assignments for $\mathrm{OH}$ stretching regions: S4: untreated wood fibre.

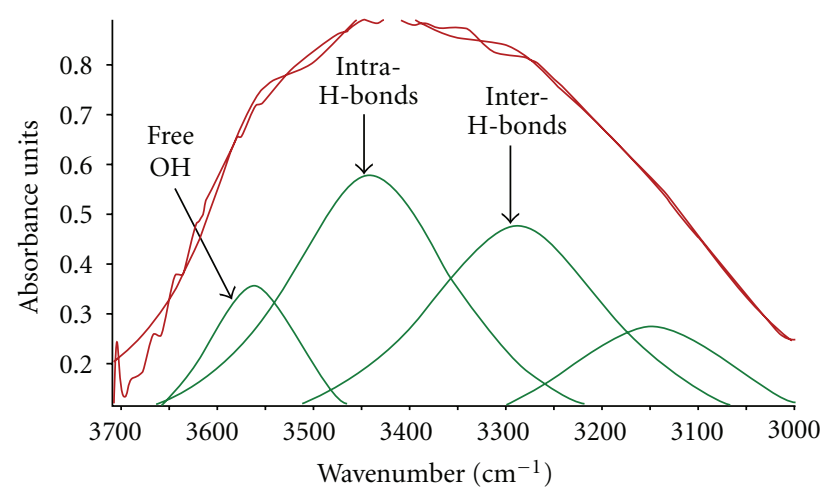

FIGURE 4: Curve fitting and peak assignments for $\mathrm{OH}$ stretching regions: S4: untreated wood fibre.

untreated fibre in the 3400 to $3100 / \mathrm{cm}$ region which is due to the intermolecular hydrogen bonds that exist between the elementary cellulose fibres showed an apparent difference in the IR absorption intensity-0.9 IR absorption units for untreated fibre compared to 0.48 IR absorption unit for biotreated fibre. This observation can be considered as a direct validation of the supposition-OS1 treatment of wood fibres can facilitate the isolation of nanofibres by reducing the intermolecular hydrogen bond density. As discussed above, the increase in internal defibrillation of the biotreated fibre can be attributed to the decrease in the intermolecular hydrogen bonds between the elementary cellulose fibres.

Intramolecular Hydrogen Bonds. The characteristic band of intramolecular hydrogen bonds in a cellulosic fibre peaks in the 3500 to $3400 / \mathrm{cm}$ region. The IR absorption intensity of the intramolecular hydrogen bond for enzyme-treated fibre showed a slight decrease compared to absorption intensity of the band for untreated fibre- -0.71 IR absorption units for untreated fibre versus 0.58 IR absorption units for enzymetreated fibres.

Free Hydroxyl Functionality. It is interesting to note that the peak $3560 / \mathrm{cm}$, characteristic of the free hydroxyl groups in the cellulose fibre, showed a slight increase in the band 
TABLE 2: Change in IR Index of $\mathrm{D}_{2} \mathrm{O}$-saturated wood fire with enzymatic treatment: OH peak from 2997 to $3724 / \mathrm{cm}$.

\begin{tabular}{lccc}
\hline $\begin{array}{l}\text { Enzyme } \\
\text { treatment }\end{array}$ & $\begin{array}{c}\text { OH peak area } \\
2997-3742 / \mathrm{cm}\end{array}$ & $\begin{array}{c}2900 / \mathrm{cm} \\
\text { Peak area }\end{array}$ & $*$ Hydrogen bonds \\
\hline S1 & 601.958 & 69.90 & 8.59 \\
S4 & 652.227 & 70.50 & 9.24 \\
\hline
\end{tabular}

S1: biotreated fibre; S4: untreated,

*Calculated by relative peak area with $2900 / \mathrm{cm}$ as internal standard.

intensity- 0.28 IR absorption units for the untreated fibre compared to 0.35 IR absorption units for the enzyme-treated fibres. This increase may be a result of the reduction in the intermolecular and the intramolecular hydrogen bonds in the cellulose fibre. The increase in free $\mathrm{OH}$ groups in cellulose fibers may not be of benefit in the context of this research as the increased hydrophilicity of these fibers can reduce their interfacial compatibility (fibre-matrix interaction) in a hydrophobic polymer matrix.

Hydrogen Bonds and Their Association. The discussion above with respect to the effect of bio-treatment on hydrogen bond density in the fiber points to the fact that the extracellular enzyme produced by the fungus OS1 has the ability to interact with the $\mathrm{OH}$ chemistry, indeed reducing the intermolecular and intramolecular hydrogen bonding.

The hydrogen bonds in a fiber may be associated with either the amorphous region or the crystalline region. In order to differentiate and understand the contribution of hydrogen bonds associated with the crystalline and the amorphous regions to the IR absorption, the biotreated and untreated fibers were saturated with deuterium $\left(\mathrm{D}_{2} \mathrm{O}\right)$, and the FT-IR absorption spectra were taken and analyzed. The hydrogen bond density, as shown in Table 2, showed a similar trend indicating the fact that the impact of biotreatment was primarily on the hydrogen bonds associated with the crystalline region of the cellulose. When the fiber is placed in an environment saturated with $\mathrm{D}_{2} \mathrm{O}$, the $\mathrm{OH}$ group associated with amorphous region will be preferentially replaced with the $\mathrm{D}_{2} \mathrm{O}$. The $\mathrm{OH}$ associated with the crystalline region of the fiber will remain unaffected due to very limited accessibility.

\section{Conclusion}

The bio-treatment of fibres has shown to have a significant positive impact on the internal defibrillation characteristics of the fibre.

(a) FT-IR absorption characteristic of the biotreated fibres has shown a marked decrease in the intermolecular hydrogen-bond $\mathrm{OH}$ functionality, while only a marginal change in intramolecular hydrogenbonded $\mathrm{OH}$ group and free $\mathrm{OH}$ groups.

(b) The fungus OS1 during its growth on fiber substrate potentially secretes enzymes capable of interacting with the hydroxyl chemistry of the fibers thereby disrupting the hydrogen bonding network. (c) The bio-treatment of fibers has the potential of being developed into an energy efficient approach for the isolation of cellulose nanofibres from plant cell wall.

\section{Acknowledgment}

The authors are grateful for the support of Natural Science and Engineering Research Council of Canada.

\section{References}

[1] F. A. L. Clowes and B. E. Juniper, Plant Cells, Blackwell Scientific Publications, 1968.

[2] C. Y. Liang and R. H. Marchessault, "Infrared spectra of crystalline polysaccharides. I. Hydrogen bonds in native celluloses," Journal of Polymer Science, vol. 37, pp. 385-395, 1959.

[3] A. Chakraborty, M. Sain, and M. Kortschot, "Cellulose microfibrils: a novel method of preparation using high shear refining and cryocrushing," Holzforschung, vol. 59, no. 1, pp. 102-107, 2005.

[4] K. Tashiro and M. Kobayashi, "Theoretical evaluation of three-dimensional elastic constants of native and regenerated celluloses: role of hydrogen bonds," Polymer, vol. 32, no. 8, pp. 1516-1526, 1991.

[5] L. A. Burglund, Cellulose Based Nanobiocomposites, CRC Press LLC, 2004.

[6] S. Janardhnan and M. Sain, "Isolation of cellulose microfibrils-an enzymatic approach," Bio-Resources, vol. 1, no. 2, pp. 176-188, 2006.

[7] W. Bolaski, A. Gallatin, and J. C. Gallatin, "Enzymatic Conversion of Cellulosic Fibers," United States Patent no. 3, 041,246, 1959.

[8] W. D. Yerkes, "Process for the digestion of cellulosic materials by enzymatic action of Trametes suaveolens," United States Patent 3, 406,089, 1985.

[9] Y. Nomura, "Digestion of pulp," 1985, Japanese Patent no. 126, 395/85.

[10] J. L. Fuentes and M. Robert, "Process of treatment of a paper pulp by an enzymic solution,” European Patent 262040, 1988.

[11] I. Uchimoto, K. Endo, and Y. Yamagishi, "Improvement of deciduous tree pulp," Japanese Patent no. 135, 1988.

[12] M. G. Paice and L. Jurasek, "Removing hemicellulose from pulps by specific enzymic hydrolysis," Journal of Wood Chemistry and Technology, vol. 4, no. 2, pp. 187-198, 1984.

[13] L. Jurasek and M. G. Paice, "Biological treatments of pulps," Biomass, vol. 15, no. 2, pp. 103-108, 1988.

[14] D. Fengel, "Characterization of cellulose by deconvoluting the $\mathrm{OH}$ valency range in the FTIR spectra," Holzforschung, vol. 46, no. 4, pp. 283-288, 1992.

[15] D. Fengel, "Influence of water on the $\mathrm{OH}$ valency range in deconvoluted FT-IR spectra of cellulose," Holzforschung, vol. 47, pp. 103-108, 1993.

[16] A. J. Michell, "Second derivative F.t.-i.r. spectra of celluloses I and II and related mono- and oligo-saccharides," Carbohydrate Research, vol. 173, no. 2, pp. 185-195, 1988.

[17] A. J. Michell, "Second-derivative F.t.-i.r. spectra of native celluloses," Carbohydrate Research, vol. 197, no. C, pp. 53-60, 1990.

[18] J. Sugiyama, J. Persson, and H. Chanzy, "Combined infrared and electron diffraction study of the polymorphism of native celluloses," Macromolecules, vol. 24, no. 9, pp. 2461-2466, 1991. 
[19] A. J. Michell, "Second-derivative FTIR spectra of native celluloses from Valonia and tunicin," Carbohydrate Research, vol. 241, pp. 47-54, 1993.

[20] J. H. Wiley and R. H. Atalla, "Band assignments in the raman spectra of celluloses," Carbohydrate Research, vol. 160, no. C, pp. 113-129, 1987.

[21] D. Gulati, Modification of interface in natural fiber reinforced composites, M.A.Sc thesis, University of Toronto, 2006. 

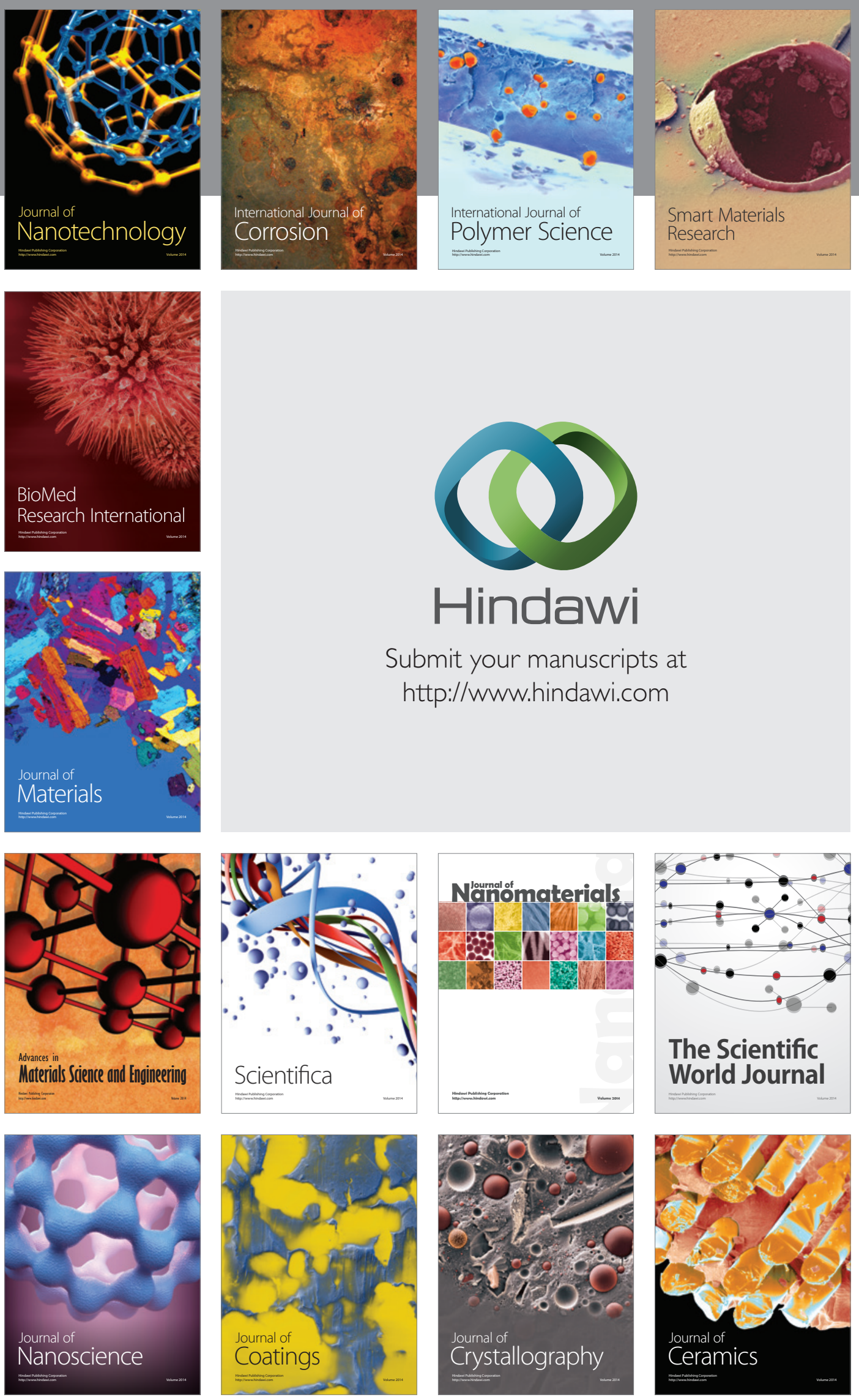

The Scientific World Journal

Submit your manuscripts at

http://www.hindawi.com

\section{World Journal}

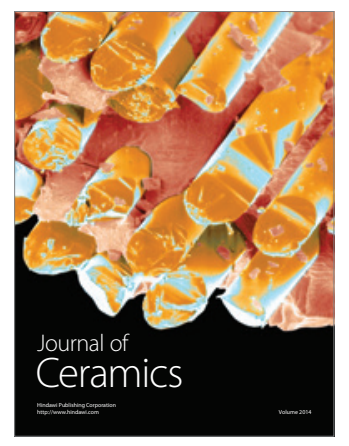

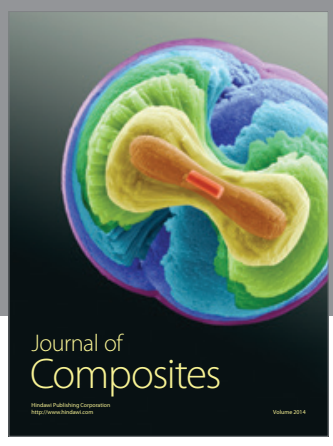
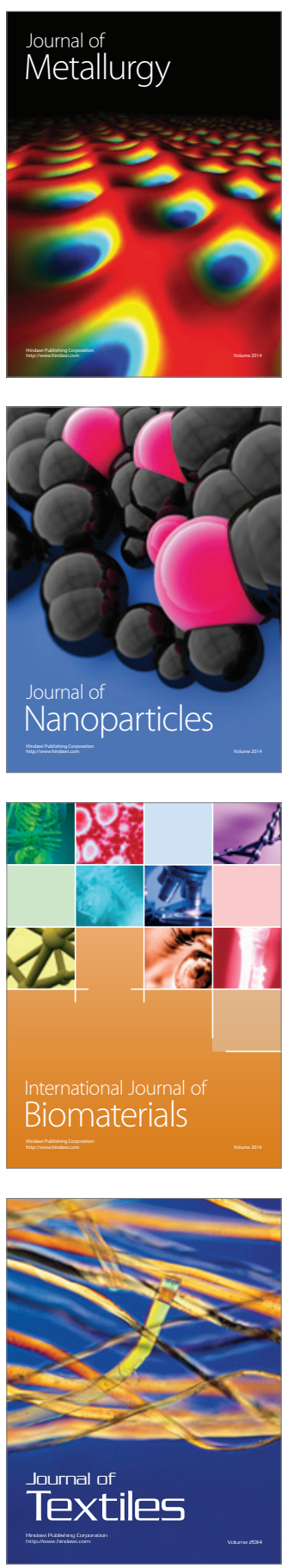